\title{
URBAN PLANNING REVOLUTION FOR INCREASING PEDESTRIAN MOBILITY IN LISBON, PORTUGAL
}

\author{
JORGE T. RIBEIRO ${ }^{1,2}$, ALEXANDRA R. VIEIRA ${ }^{3}$, SUSANA ROSADO $^{1}$ \& FRANCISCO SERDOURA ${ }^{1,3}$ \\ ${ }^{1}$ Lisbon School of Architecture, University of Lisbon, Portugal \\ ${ }^{2}$ CERENA, University of Lisbon, Portugal \\ ${ }^{3}$ CIAUD, University of Lisbon, Portugal
}

\begin{abstract}
This paper focuses on the change in terms of spatial structure of the old cities with the urban renewal. The connection of places in the city through architecture and urbanism, encouraging walkability, will strengthen intergenerational and social interactions, will also reinforce cultural expressions, and provide social cohesion and security of the societies, as quoted in Habitat III - for better urban future. The permeability and connection between the different parts of the block, the street and the buildings will create links between space, shape and use will offer its users an increase in the quality of life, minimizing the use of motorized vehicles. The idea is to implement urban solutions that guarantee greater walkability by simulating an increase of passages in a neighborhood of Lisbon, Portugal. To test the feasibility of this idea, graph theory, space syntax, and data analysis methods and techniques were used to create several scenarios for increasing the pedestrian mobility in Lisbon. As results in all the three hypothetical scenarios created - increasing in number of passages and alternatives in a shortterm (Simulation 1), medium-term (Simulation 2) and long-term (Simulation 3) intervention logic there were somehow improvements in terms of walkability and accessibility. As the passages through the interior of the blocks increase, the network is also more easily accessible. The proposed research confirms that urban passages (e.g., the pasáž in Prague) are essential for changing the walkability paradigm in old cities rehabilitation planning.

Keywords: pedestrian mobility, accessibility, passages, walkability, normalized angular choice, normalized angular integration, to-movement, through-movement, urban planning, Bairro dos Actores.
\end{abstract}

\section{INTRODUCTION}

Focusing on the cities, which are expected to be occupied by two-thirds of the world's population by 2050 [1] and whose urban areas produce $70 \%$ of the emissions that are propelling the planet to an unknown climate [2] there is an urgent need to change the paradigm of the planet's population life by rethink and reimagining [2] the cities. At the same time, this becomes an obvious need and challenge. In this context, Otto [2] defines this need as a "planning revolution" accomplished by generating "compact cities strategically structured with neighborhoods and mixed-use buildings and with an emphasis on integrated urban systems."

The monofunctional and dispersed city, criticized by Alexander [3] led to the discovery of innovative ideas in processes of analysis and the urban design of forms in architecture; establishing a list of standards that provide guidelines for urbanism and architecture; where the graphs' theory was essential to find mathematical solutions for architecture and urban planning.

The exploratory research presented in this article seeks to incorporate this context. In old and consolidated cities, it is necessary to increase their permeability to encourage the walkability of their inhabitants. Inspired by the network communications, namely regarding the concepts of connection, communication, sharing, and exchange, it was then thought of creating passages that would open closed blocks, establishing connections between streets 

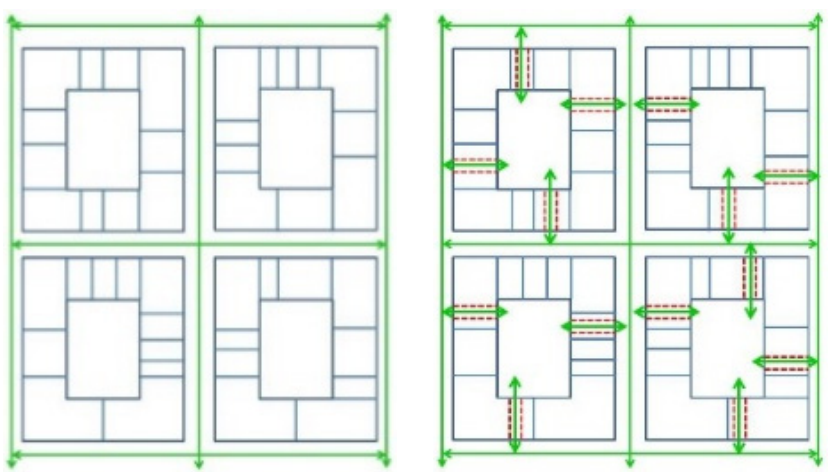

Figure 1: Schematic idea to open closed blocks using passages.

that enable pedestrian movements (Fig. 1). In other words, given a study area that includes a neighborhood with blocks with unused interior spaces, with buildings and shops that are degraded, abandoned or under renovations, different situations by opening of passages were created that would arise in these points of easy intervention.

In particular, the exploratory analysis aims to achieve improvements in terms of accessibility and spatial structure when the number of passages between existing streets is increased.

The hypothesis of this exploratory analysis is to assess whether the passages theoretically improve spatial structure and accessibility. Three hypothetical scenarios are created, and the respective simulations are performed in terms of measures under analysis. These scenarios are increasing in terms of number of passages and alternatives in a shortterm (Simulation 1), medium-term (Simulation 2) and long-term (Simulation 3) intervention logic.

To test the feasibility of this idea, graph theory, space syntax, and data analysis methods and techniques and an historic neighborhood of Lisbon were used to simulate several scenarios for increasing the pedestrian mobility in Lisbon.

This approach will meet the Sustainable Development Goals of Good Health and WellBeing (SDG3) since people will walk more using those pedestrian paths in their dally-life bases; also, this involves a Climate Action (SDG13) as there would be a significant reduction of the use of cars, buses, and taxis; all these actions towards Sustainable Cities and Communities (SDG11).

This paper consists of six sections. After the Introduction, Section 2 presents a brief literature review about the context and the main tools. Section 3 presents the case study in Lisbon. The materials and methods used in simulations and the main results as its discussion are presented, respectively, in Sections 4 and 5. Section 6 presents the main conclusions.

\section{LITERATURE REVIEW}

The interdisciplinary knowledge about the cities is now, more than ever, a very important tool to increase the citizens' quality of life.

The relationship of systems and concepts resulting from architecture, economics, sociology, technology and urbanism, will allow the construction of cities without borders and with a predominance of open spaces, greater mobility, and biodiversity, where people will be able to walk in the city for periods of 15 minutes, called "hyperconnectivity" or 
"chrono-urbanism" as defined by the authors of [4] and [5]. The same idea, that the city should be experienced through pedestrian displacement [6], [7], considering the valorization of public space and the relationship that is established between buildings was also mentioned in [8]. The balance between urban architecture and man is notorious for harmonizing the experience of qualified public spaces, providing cities that are alive, safe, sustainable, and healthy for the citizens. The relationships between the built space (urban and architectural) and social behavior result from the establishment of guidelines achieved between architecture and urbanism; this means the relationship of the human scale achieved, and the sociological principles applied [9], [10]. The human scale can be represented in the city planning if considered by different scientific areas, starting from the particular to the general; working together on the small scale of the city, such as the street or the block, which in turn will influence the areas of closeness in a useful and consolidated way. The definition and establishment of city uses will create interconnections, depending on how the people felt the experience lived in these places, good or bad. The buildings' design will be relevant [11] for gathering information about the urban space that will directly influence the way the pedestrian uses the public space [5], [12]. Ascher [13] studied the sociological point of view, the urban mobility and the dynamics of life-andurbanization concerning to the modern cities and concluded that they are essential for cities to become alive and dynamic.

Considering the evolution of the functions of the city over time, from the modern city to post-modernity, the phenomenon of megalopolis was reached, as explored by Freitag [14]. The demographic growth and the territorial extension of the cities will cause the loss of cultural and architectural/urban references that are part of their societies, critically influencing the social, economic, and humanist actions that should be mitigated through sustainable, safe walkability and sociable, that can effectively connect people, places, goods, and services, providing a balanced and integrated development either architecturally or socially. Cities are expected to be safe, healthy, accessible, resilient, and sustainable places, providing, and fostering prosperity and quality of life for those who live or use these places. The connection of places in the city through architecture with urbanism, encouraging walkability, will strengthen intergenerational and social interactions, will also reinforce cultural expressions, and provide social cohesion and security of the societies, as quoted in Habitat III - for better urban future - Declaration of Quito [15]. The permeability and connection between the different parts of the block, the street and the buildings will create links between space, shape and use will offer its users an increase in the quality of life, minimizing the use of motorized vehicles and consequently an increase in the preservation of biodiversity within cities. So, it is essential to study the relationship between space and society in its different aspects.

Space syntax allows for analysis of space combining movement and land use factors with cognition and behavior [16]. It only considers how each space is related to all other spaces in terms of directional changes and angular deviation within a spatial system [17]. Space syntax measures to-movement (or closeness) and through-movement (or betweenness) potentials of every public street segment in relation to all other segments [18]. According to Hillier et al. [6] and Penn et al. [19], space syntax's "theory of natural movement" is a useful tool for comparing "before" and "after" conditions of spatial changes that result from restructuring (or simulating new situations). The theory of the natural movement states that the flow of human movement in built environments depends on the degree of spatial integration (i.e., normalized distance from one point to all points [9]) of the street network. The higher spatial integration on various scale levels, the higher the flow of human movement [19]. 
For more than 30 years, the axial map has constituted the basis of the Space Syntax. However, from the beginning of the 21st century, angular segment analysis by metric distance becomes a more powerful tool [20]. According to Yamu et al. [16], the solution for successful computational angular analysis with various local radii were to break down the axial map into a segment map and incorporate segment length into the calculations [21]. This operation can be performed with the Depthmap software.

So, according to De Koning et al. [22], the measures within space syntax that best capture spatial configurations are [9] the potential to-movement which measures how likely a street is to be a destination of a route - the angular segment integration analyses (i.e., how close each segment is to all others in terms of the sum of angular changes that are made on each route); and the potential through-movement which measures how likely a street is used as part of a route-the angular choice analysis (i.e., counting the number of times each street segment falls on the shortest path - least angular deviation between all pairs of segments within a selected distance or radius). In Hillier et al. [18] a normalization of the measures of angular choice and angular integration is suggested for better properties of the measures. These measures will be referred to as normalized angular choice (NACH) and normalized angular integration (NAIN).

According to Orsini [23] the most important variables calculated by the angular segment analysis are choice and integration and while integration calculates the distance between all origin and destination spaces in a system (closeness), choice, known as betweenness centrality in graph theory, deals with the decision-making process and highlights which itinerary is preferred to move anywhere in the study area. Choice quantifies the flow passing through each space, by summing all nodes located on the shortest paths joining any destination from an origin space. That is why the measure must be normalized as the nodes located in segregated areas have total and average values higher than the nodes located in integrated areas. Choice values of each segment are divided by their total depth [18].

Considering the foreground and background network, according to Orsini [23], its robustness can be assessed with the angular normalization of both integration and choice variables. The two measures provide information on the spatial performance and morphological properties of the study area. NACH allows understanding the spatial structure of cities on foreground (maximum value) and on background (mean value) while NAIN indicates how both foreground (Max) and background (Mean) networks are easily accessible. The potential to and through-movement in the background network is represented by Mean values of NACH and NAIN and by Maximum NACH and NAIN indexes in the foreground network [24].

\section{CASE STUDY}

The need for expansion of the city of Lisbon, in the first decades of the 20th century, forced a rethink and introduced changes from an urban point of view, which led to its growth to the North, predominantly rural at the time. The General Plan for Urbanization and Expansion of Lisbon (PGUEL) of 1938-1948, would define new programmatic and architectural areas that aimed to represent a new way of living in Lisbon society.

Actores neighborhood (Bairro dos Actores) appears in the 1930s, as an integral part of PGUEL, and because of the lack of housing in the city and the desire to assert a nationalist political regime at the time [25], [26]. With an area of approximately 14 ha, it is limited to the west by Almirante Reis Avenue (Avenida Almirante Reis) and to the south by Dom Afonso Henriques Avenue (Alameda Dom Afonso Henriques) (Fig. 2). 


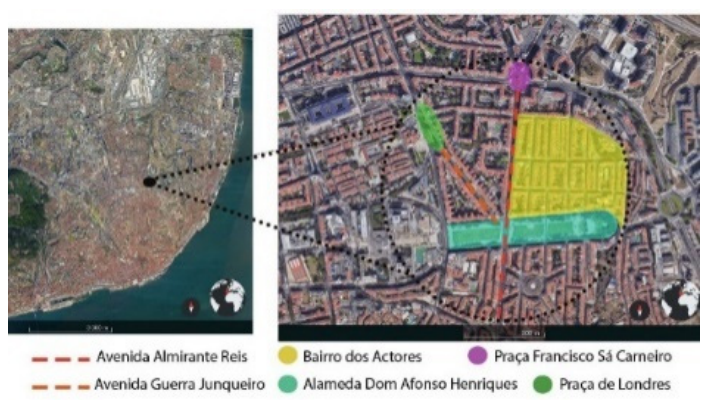

Figure 2: Bairro dos Actores location in Lisbon.

The organization of facilities and the road hierarchy are the main innovations to be taken into account in this urban project for the city of Lisbon. The articulation between the different urban elements also contributed to the morphological and social homogeneity that defined the neighborhood units.

This part of the city is defined by four streets perpendicular to the structural road axisAlmirante Reis Avenue-which, in turn, intersect with other streets, creating an orthogonal standard metric between the blocks of the neighborhood.

The urban configuration of Bairro dos Actores allows for a direct relationship with its uses, allowing an intelligible walk throughout the neighborhood similar to the pedestrian route length.

There are some spaces belonging to these buildings, predominantly on the ground floor, vacant awaiting rehabilitation, and interventions in terms of uses and functions to adjust to the new needs of the neighborhood and greater urban mobility.

The relationship between urban form and its use, allows this place to become safe, inclusive, and resilient, and existing spatial configurations must be reconsidered, solving urban spatial conflicts, and creating attractiveness and well-being for those who live and travel through this neighborhood.

\section{MATERIALS AND METHODS}

For computing the space syntax analysis for the different simulations, basic maps were manually generated for the study area. The axial map was drawn on the city of Lisbon digital maps at a scale of 1:1,000 so that the location of the passages in the neighborhoods' blocks can be defined as accurately as possible. Considering the study scale and size of Bairro dos Actores neighborhood, to reduce the impact of edge effects the axial lines have been drown for a buffer area of 1,500 m, which corresponds to an average walking time of approximately 30 minutes (Fig. 3).

According to Yamu et al. [16] the solution for successful computational angular analysis with various local radii were to break down the axial map into a segment map (generated by breaking the original axial lines at their intersections) and incorporate segment length into the calculations [21]. And so, the axial map of Bairro dos Actores was transformed into a segment map (DepthmapX software, version 0.80 was used) to obtain more clearly the importance of the proposed passages to identify the increase/improvement of walkability within Bairro dos Actores.

The segment map allows for calculation and measures of different walkability possibilities in the existing and proposed street system for the neighborhood. 

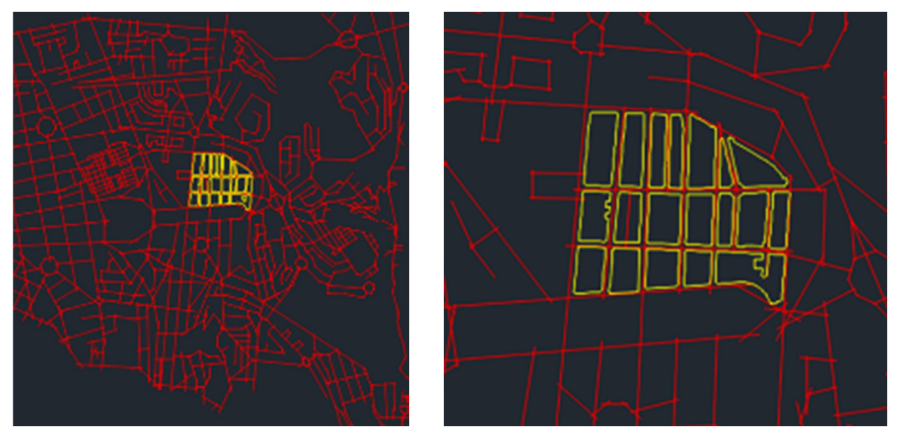

Figure 3: Axial map (red lines) and neighborhood structure (yellow lines).

According to De Koning [22], the measures within space syntax that best capture spatial configurations are [9] the potential to-movement which measures how likely a street is to be a destination of a route - the angular segment integration analyses (i.e., how close each segment is to all others in terms of the sum of angular changes that are made on each route); and the potential through-movement which measures how likely a street is used as part of a route-the angular choice analysis (i.e., counting the number of times each street segment falls on the shortest path - least angular deviation between all pairs of segments within a selected distance or radius). In Hillier et al. [18] a normalization of the measures angular choice and angular integration is suggested for better properties of the measures. These measures will be referred to as normalized angular choice (NACH) and normalized angular integration (NAIN).

This study focuses on the analysis of those two syntactic measures: NACH and NAIN. From the spatial analysis to the Bairro dos Actores, it was felt that the two syntactic measures, mentioned above, can better explain the potential to generate pedestrian movements, considering the possibilities of proposing new passages in the blocks in that urban area.

\section{RESULTS}

The use of Depthmap software allowed the angular computation of the NAIN and NACH segment map with three local radii: $250 \mathrm{~m}, 400 \mathrm{~m}$, and $750 \mathrm{~m}$. In addition to the $400 \mathrm{~m}$ and $750 \mathrm{~m}$ metric radii corresponding to 5 minute and 10 minute walk, respectively [16], it was also chosen to use the $250 \mathrm{~m}$ metric radii. In fact, given that the case study is part of a parish with a high aging rate (208.1 people aged 65 and over for every 100 people under 15 years old [27]), it is considered that the $250 \mathrm{~m}$ correspond to 5 minute walk for the elderly population.

Fig. 4 shows the NAIN results for the global area.

Globally and in any of the metric radii, there is an increase in NAIN as the number of passages increases, except in Simulation 3 (when the number of passages is the largest of those tested). Most likely, the study area acquires a too high leading role, becoming an attraction; this has the consequence of "drying up" the surrounding area and leads to the conclusion that the increase of connections is not enough only in a neighborhood, but it should be done in a wider area to increase global and not just local walkability.

Since NAIN measures the potential to-movement, i.e. how likely a street is to be a destination of a route, the increase of passages in Bairro dos Actores tends to attract the routes to that study area. 


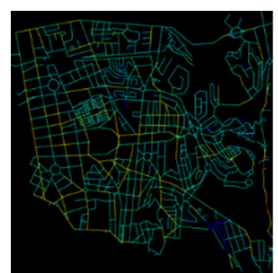

$250 \mathrm{~m}$

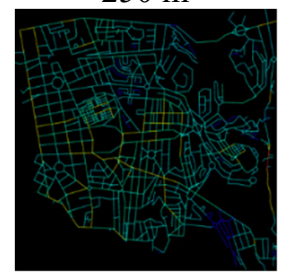

$400 \mathrm{~m}$

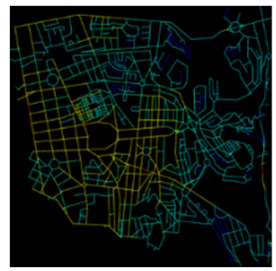

$750 \mathrm{~m}$

Current

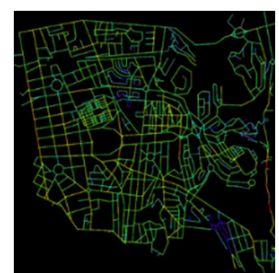

$250 \mathrm{~m}$

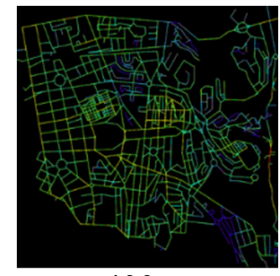

$400 \mathrm{~m}$

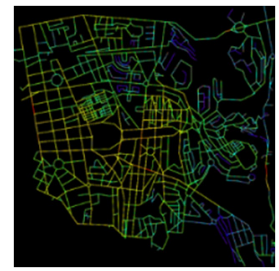

$750 \mathrm{~m}$

Simulation 1

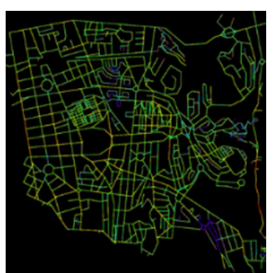

$250 \mathrm{~m}$

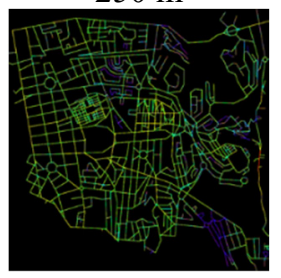

$400 \mathrm{~m}$

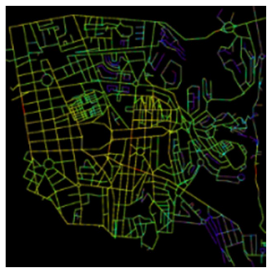

$750 \mathrm{~m}$

Simulation 2

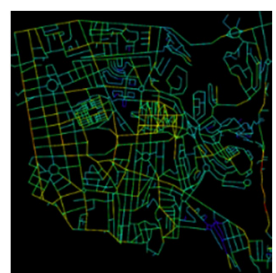

$250 \mathrm{~m}$

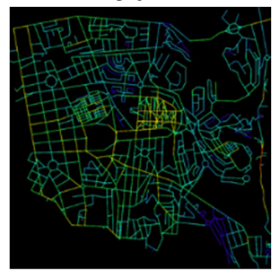

$400 \mathrm{~m}$

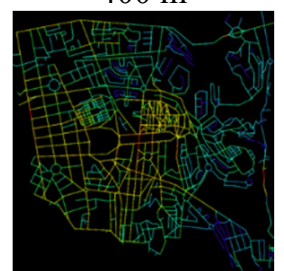

$750 \mathrm{~m}$

Simulation 3

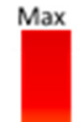

Figure 4: NAIN global map of current situation and three simulations.

In shorter metric radii $(250 \mathrm{~m}-5$ minute walk for the elderly population and $400 \mathrm{~m}-$ 5 minute walk) there are no major differences in NAIN in any of the simulations considered, while in the larger metric radii (750 $\mathrm{m}$ - 10-minute walk) there is a considerable increase in the NAIN in areas with more regular urban grids.

Fig. 5 and Table 1 show the NAIN results for the study area.

Analyzing the maps of Fig. 5 there appears to be an increase of NAIN values across all metric radii. For a more accurate analysis, the NAIN values were quantitatively analyzed in terms of their variation (standard deviation), mean and maximum value.

Both in the current situation and in the three simulations, the standard deviation (SD) is reduced and identical in any of the radii, revealing results homogeneity. The to-movement potential [16] of the spatial structure on background (mean value) and on foreground (maximum value) [23] is always increasing. That is, as the passages through the interior of the blocks increase, the network is also more easily accessible [23], in all metric radii (Fig. 6). It also highlights that the smaller metric radii $(250$ and $400 \mathrm{~m})$ present NAIN values higher than the corresponding values for the larger metric radii $(750 \mathrm{~m})$, revealing that the to-movement potential is greater in the smaller distances (Fig. 6).

Fig. 7 shows the NACH results for the global area.

Globally and in any of the metric radii, there is an increase in NACH as the number of passages in Bairro dos Actores increases, essentially in Simulations 2 and 3, with no evidence of the same effect detected in the case of NAIN. 


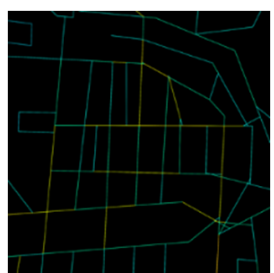

$250 \mathrm{~m}$

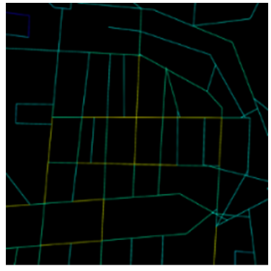

$400 \mathrm{~m}$

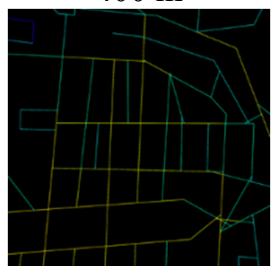

$750 \mathrm{~m}$

Current

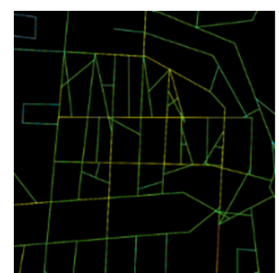

$250 \mathrm{~m}$

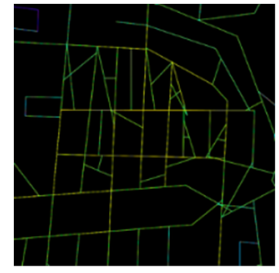

$400 \mathrm{~m}$

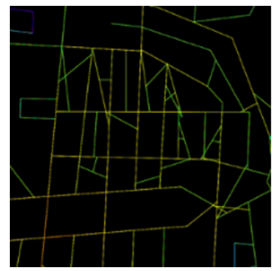

$750 \mathrm{~m}$

Simulation 1

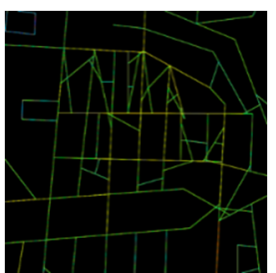

$250 \mathrm{~m}$

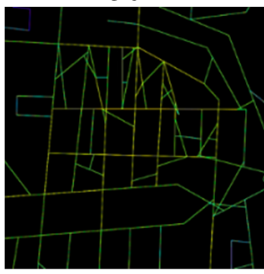

$400 \mathrm{~m}$

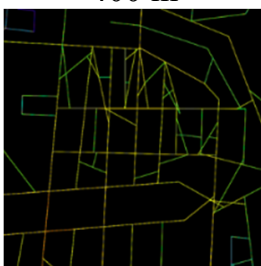

$750 \mathrm{~m}$

Simulation 2

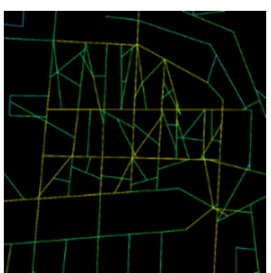

$250 \mathrm{~m}$

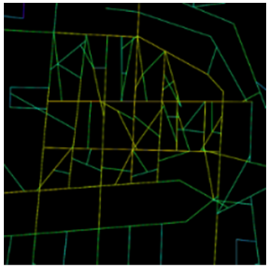

$400 \mathrm{~m}$

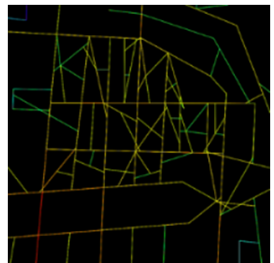

$750 \mathrm{~m}$

Simulation 3

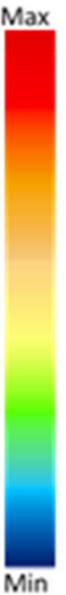

Figure 5: NAIN neighborhood map of current situation and three simulations.

Table 1: NAIN results of current situation and three simulations.

\begin{tabular}{|l|c|c|c|c|c|c|c|c|c|c|c|c|}
\hline & \multicolumn{3}{|c|}{ Current } & \multicolumn{3}{c|}{ Simulation 1 } & \multicolumn{3}{c|}{ Simulation 2 } & \multicolumn{3}{c|}{ Simulation 3 } \\
\cline { 2 - 13 } & Mean & Max & SD & Mean & Max & SD & Mean & Max & SD & Mean & Max & SD \\
\hline NAIN250 & 1.624 & 2.128 & 0.241 & 1.646 & 2.244 & 0.285 & 1.636 & 2.244 & 0.289 & 1.702 & 2.341 & 0.284 \\
\hline NAIN400 & 1.525 & 1.965 & 0.220 & 1.603 & 2.191 & 0.270 & 1.613 & 2.259 & 0.283 & 1.717 & 2.420 & 0.283 \\
\hline NAIN750 & 1.224 & 1.562 & 0.129 & 1.298 & 1.647 & 0.154 & 1.329 & 1.672 & 0.166 & 1.440 & 1.810 & 0.176 \\
\hline
\end{tabular}
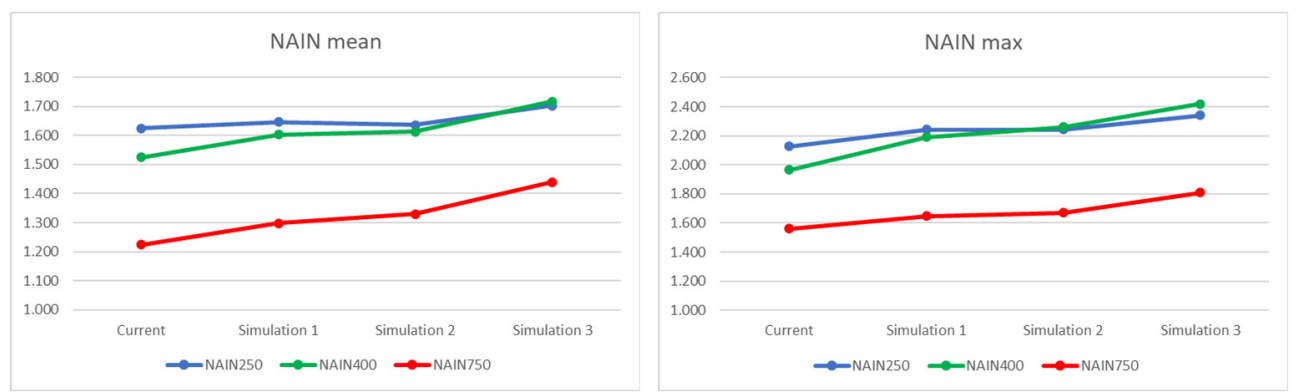

Figure 6: Evolution of NAIN (mean and max) in the different situations and metric radii. 


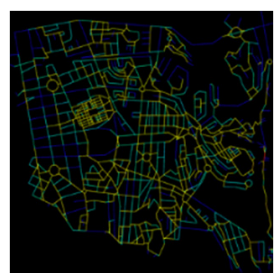

$250 \mathrm{~m}$

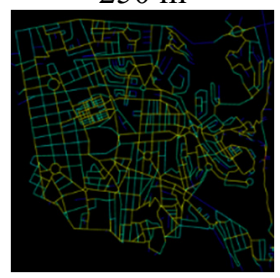

$400 \mathrm{~m}$

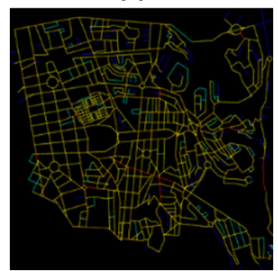

$750 \mathrm{~m}$

Current

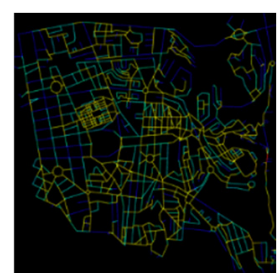

$250 \mathrm{~m}$

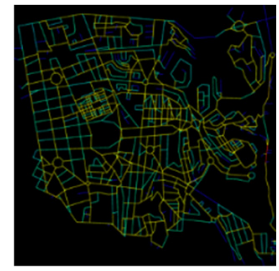

$400 \mathrm{~m}$

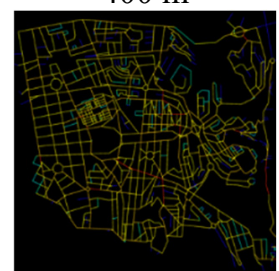

$750 \mathrm{~m}$

Simulation 1

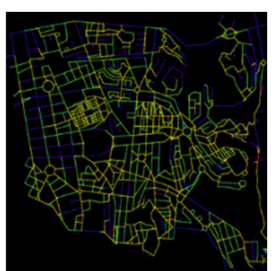

$250 \mathrm{~m}$

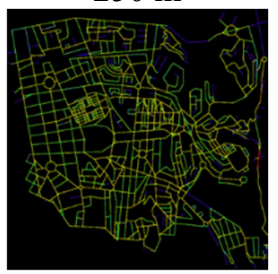

$400 \mathrm{~m}$

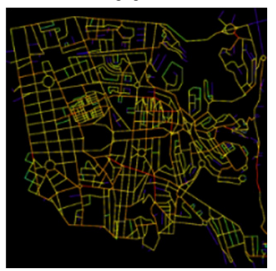

$750 \mathrm{~m}$

Simulation 2

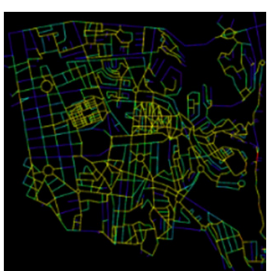

$250 \mathrm{~m}$

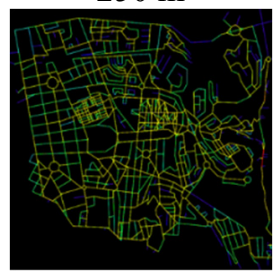

$400 \mathrm{~m}$

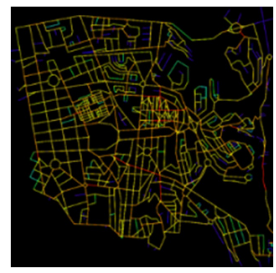

$750 \mathrm{~m}$

Simulation 3

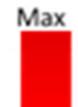

Min

Figure 7: NACH global map of current situation and three simulations.

In shorter metric radii $(250 \mathrm{~m}-5$ minute walk for the elderly population and $400 \mathrm{~m}-$ 5 minute walk) there are no major differences in NACH in any of the simulations considered, while in the larger metric radii $(750 \mathrm{~m}-10$ minute walk) there is a considerable increase in the NACH.

Fig. 8 and Table 2 show the NACH results for the study area.

Analysing Fig. 8, it appears to be a greater increase of NACH values in the $750 \mathrm{~m}$ metric radii. Also, in this measure NACH values were quantitatively analysed in terms of its variation (standard deviation), mean and maximum value.

The homogeneity of the NACH results is also verified, expressed by the low and identical values of the standard deviation (SD) in the three simulations and in all metric radii. The through-movement potential [16] of the spatial structure on background (mean value) and on foreground (maximum value) [23] increases with the increase of passages through the interior of the blocks in almost all metric radii (Fig. 9). The only exception is a slight decrease of the NACH_400 max in Simulation 3 (Fig. 9(b)), i.e. the throughmovement potential [16] of the spatial structure on foreground (maximum value) does not necessarily increase whenever there is a greater number of passages through the blocks.

By comparison, the study carried out in Orsini [23] shows how NAIN and NACH measures also allow analyzing walkability in an evolutionary way due to changes in urban morphology (the increasing number of passages in this paper). 


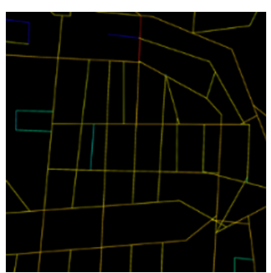

$250 \mathrm{~m}$

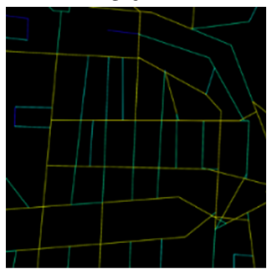

$400 \mathrm{~m}$

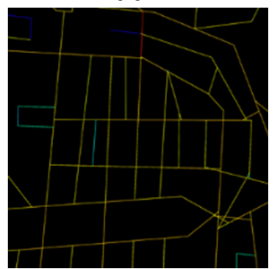

$750 \mathrm{~m}$

Current

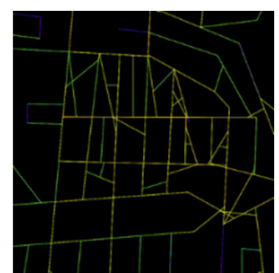

$250 \mathrm{~m}$

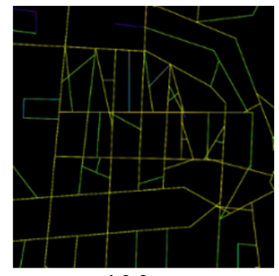

$400 \mathrm{~m}$

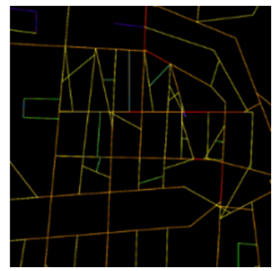

$750 \mathrm{~m}$

Simulation 1

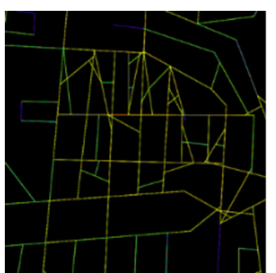

$250 \mathrm{~m}$

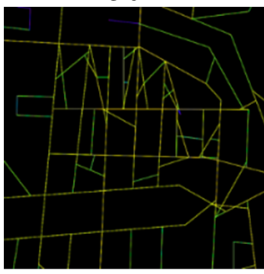

$400 \mathrm{~m}$

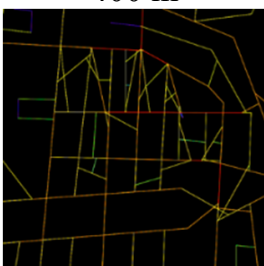

$750 \mathrm{~m}$

Simulation 2

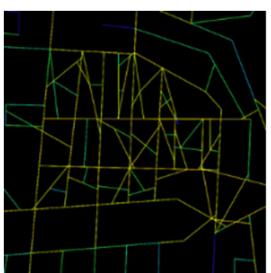

$250 \mathrm{~m}$

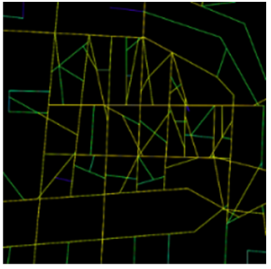

$400 \mathrm{~m}$

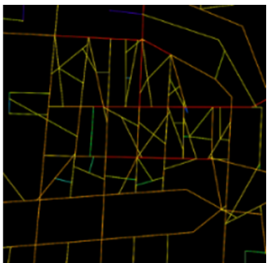

$750 \mathrm{~m}$

Simulation 3

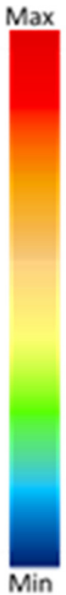

Figure 8: NACH neighborhood map of current situation and three simulations.

Table 2: NAIN results of current situation and three simulations.

\begin{tabular}{|c|c|c|c|c|c|c|c|c|c|c|c|c|}
\hline & \multicolumn{3}{|c|}{ Current } & \multicolumn{3}{|c|}{ Simulation 1} & \multicolumn{3}{|c|}{ Simulation 2} & \multicolumn{3}{|c|}{ Simulation 3} \\
\hline & lean & Max & SD & Mean & Max & $\mathrm{SD}$ & Mean & $\operatorname{Max}$ & $\mathrm{SD}$ & Mean & Max & SD \\
\hline & & & & 44 & & & & & 0227 & & & 0.237 \\
\hline & & & & 776 & & & 084 & & & 1.094 & & 245 \\
\hline ACH750 & .056 & 1.291 & 0.210 & 1.060 & 1.343 & 0.248 & 1.072 & 1.356 & 0.235 & 1.089 & 1.427 & 0.242 \\
\hline
\end{tabular}

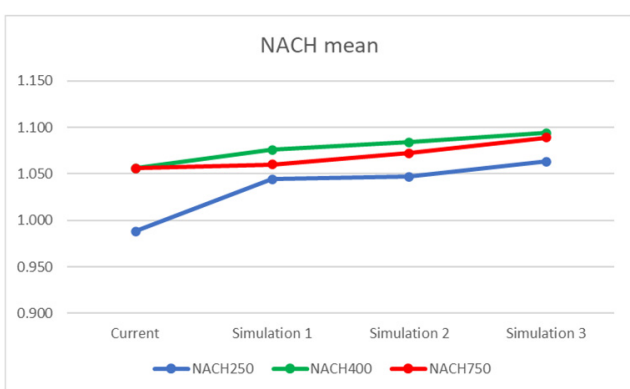

(a)

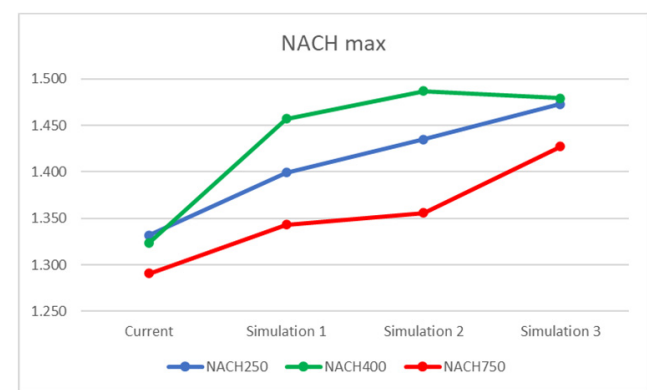

(b)

Figure 9: Evolution of NACH (mean and max) in the different situations and metric radii. 


\section{CONCLUSIONS}

With this exploratory analysis we were able to accept the hypothesis that the increase in the number of passages improved spatial structure and accessibility of the study area. In all the three hypothetical scenarios created - increasing in number of passages and alternatives in a short-term (Simulation 1), medium-term (Simulation 2) and long-term (Simulation 3) intervention logic - there were somehow improvements in terms of walkability and accessibility.

The to-movement potential (which measures how likely a street is to be a destination of a route) of the spatial structure on background (mean value) and on foreground (maximum value) is always increasing. That is, as the passages through the interior of the blocks increase, the network is also more easily accessible, in all metric radii.

The through-movement potential (which measures how likely a street is used as part of a route) of the spatial structure on background (mean value) and on foreground (maximum value) increases with the increase of passages through the interior of the blocks in almost all metric radii, except in Simulation 3 (metric radii $400 \mathrm{~m}$ ) where there was a slight decrease, so the through-movement potential of the spatial structure on foreground (maximum value) does not necessarily increase whenever there is a greater number of passages through the blocks.

In old and consolidated cities this idea of increasing their permeability to encourage the walkability of their inhabitants should be easy to implement if the opening of new passages were considered in the building rehabilitation plans. Simulations like the ones performed in this paper would be extremely relevant to access the feasibility of the new possible passages.

The proposed research confirms that Urban Passages play a crucial role in changing the walkability paradigm in old cities rehabilitation planning.

\section{REFERENCES}

[1] United Nations, Department of Economic and Social Affairs, Population Division. World Urbanization Prospects: The 2018 Revision (ST/ESA/SER.A/420), United Nations: New York, 2019.

[2] United Nations Environment Programme, Cities of the Future: The Ultimate Design Challenge. https://www.unep.org/news-and-stories/story/cities-future-ultimatedesign-challenge. Accessed on: 10 Jul. 2019.

[3] Alexander, C., A city is not a tree. Architectural Forum, 122(1), pp. 58-62, 1965.

[4] Moreno, C., The 15 Minutes City: For a New Chrono-Urbanism! http://www.moreno-web.net/the-15-minutes-city-for-a-new-chrono-urbanism-prcarlos-moreno/. Accessed on: 15 Mar. 2020.

[5] Moreno, C., Allam, Z., Chabaud, D., Gall, C. \& Pratlong, F., Introducing the "15minute city": Sustainability, resilience and place identity in future post-pandemic cities. Smart Cities, 4(1), pp. 93-111, 2021. DOI: 10.3390/smartcities4010006.

[6] Hillier, B., Penn, A., Hanson, J., Grajewski, T. \& Xu, J., Natural movement: Or configuration and attraction in urban pedestrian movement. Environment and Planning B, 20(1), pp. 29-66, 1993. DOI: 10.1068/b200029.

[7] Koohsari, M.J., Oka, K., Owen, N. \& Sugiyama, T., Natural movement: A space syntax theory linking urban form and function with walking for transport. Health and Place, 58(102072), 2019. DOI: 10.1016/j.healthplace.2019.01.002.

[8] Gehl, J., Life Between Buildings: Using Public Space, Island Press, 2011.

[9] Hillier, B. \& Hanson, J., The Social Logic of Space, Cambridge University Press: Cambridge, 1984. 
[10] Ferwati, M., Controllability of traditional neighborhood and its simplified layout. Archnet-International Journal of Architectural Research, 4(1), pp. 75-84, 2010.

[11] Vieira, A.R. \& Ribeiro, J.T., Network buildings: Definition and assessment. IOP Conference Series: Materials Science and Engineering, 603(022070), pp. 25-32, 2019. DOI: $10.1088 / 1757-899 X / 603 / 2 / 022070$.

[12] Lynch, K., A Boa Forma da Cidade [The Good Shape of the City], Edições 70: Lisbon, 2010.

[13] Ascher, F., Novos Princípios do Urbanismo: Seguido de Novos Compromissos Urbanos [New Principles of Urbanism: Followed by New Urban Commitments], Livros Horizonte: Lisbon, 2010.

[14] Freitag, B., Teorias da Cidade [City Theories], Papirus: Campinas, 2006.

[15] United Nations-Habitat III, Nova Agenda Urbana [New Urban Agenda]. https://uploads.habitat3.org/hb3/NUA-Portuguese.pdf. Accessed on: 15 Mar. 2020.

[16] Yamu, C., Van Nes, A. \& Garau, L., Bill Hillier's legacy: Space syntax - A synopsis of basic concepts, measures and empirical application. Sustainability, 13(6), pp. 3394, 2021. DOI: 10.3390/su13063394.

[17] Hillier, B., Space as paradigm for understanding strongly relational systems. Proceedings of the 2nd International Space Syntax Symposium, pp. 56.1-56.16, 1999.

[18] Hillier, B., Yang, T. \& Turner, A., Normalising least angle choice in Depthmap and how it opens new perspectives on the global and local analysis of city space. Space Syntax, 3(2), pp. 155-193, 2012.

[19] Penn, A., Hillier, B., Banister, D. \& Xu, J., Configurational modelling of urban movement network. Environment and Planning B, 25(1), pp. 59-84, 1998.

DOI: $10.1068 / \mathrm{b} 250059$.

[20] Carmona, M., Explorations in Urban Design: An Urban Design Research Primer, Ashgate Publishing: Farnham, 2014.

[21] Hillier, B., The theory of the city as object: Or how spatial laws mediate the social construction of urban space. Proceedings of the 3rd International Space Syntax Symposium, pp. 02.1-02.28, 2001.

[22] De Koning, R., Tan, W. \& Van Nes, A., Assessing spatial configurations and transport energy usage for planning sustainable communities. Sustainability, 12(19), 8146, 2020. DOI: 10.3390/su12198146.

[23] Orsini, H.F., Belgrade's urban transformation during the 19th century: A space syntax approach. Geographica Pannonica, 22(3), pp. 219-229, 2018.

DOI: $10.5937 /$ gpp22-18026.

[24] Hillier, B., Yang, T. \& Turner, A., Advancing Depthmap to advance our understanding of cities: Comparing streets and cities and streets to cities. Proceedings of the 8th International Space Syntax Symposium, pp. 135-142, 2012.

[25] Fernandes, J.M., Lisboa no século XX: O tempo moderno [Lisbon in the 20th century: the modern time]. O Livro de Lisboa [The Book of Lisbon], Livros Horizonte: Lisbon, pp. 493-522, 1994.

[26] França, J.A., Lisboa: Urbanismo e Arquitectura [Lisbon: Urbanism and Architecture], Livros Horizonte: Lisbon, 1980.

[27] Junta de Freguesia do Areeiro, População [Population]. https://jf-areeiro.pt/ populacao-2/. Accessed on: 26 Jun. 2021. 